\title{
A Passive Network Synthesis from Two-Terminal Measured Impedance Characteristic
}

\author{
David KROLAK, Pavel HORSKY \\ ON Design Czech, s.r.o., Videnska 204/125, 61900 Brno, Czech Republic \\ \{David.Krolak, Pavel.Horsky\}@onsemi.com \\ Submitted October 22, 2018 / Accepted January 2, 2019
}

\begin{abstract}
A linear high-frequency lumped-element model extraction from a two-terminal measured impedance by an improved element-by-element extraction method is described. This extraction method is extended to series and shunt resistors extraction as lossy elements of passive (linear) circuit network. The extracted linear circuit model from the two-terminal impedance of ideal and realistic passive networks is validated by SPICE simulations in a frequency domain. The extracted model can be used for more accurate electrical environment modeling and SPICE simulations of integrated circuits including external passive networks. An example of passive network synthesis from the two-terminal measured impedance by a proposed software is also presented.
\end{abstract}

\section{Keywords}

Element-by-element extraction, EMC, impedance measurement, lumped-element model, passive network synthesis, transmission line

\section{Introduction}

With the increasing advent of high speed and radio frequency (RF) mixed signal devices, maintaining a signal integrity and an electromagnetic interference (EMI) have become one of major issues facing a modern integrated circuit design. The EMI such as an electromagnetic susceptibility (EMS) of integrated circuit (IC) is often given by an IC environment. The IC environment is considered such as an electric impedance of printed circuit board (PCB) tracks with electrical components at the IC pins. An RF direct power injection (DPI) method to the dedicated IC pin described in [1] is used for the EMS testing at IC level. An equivalent circuit representation of RF DPI coupling path to the IC pin is a vital task for the EMS simulation of IC.

Passive network synthesis from required circuit function is a classical subject in the electrical circuit theory which was researched in a "golden era" from the 1930s to the 1970s, for example [2] and [3]. Nowadays modeling of passive network elements according to high frequency
S-parameters measurements are developed. For example, [4] describes a method using S-parameters for circuit modeling of multi-layer ceramic capacitors. This method is successfully used by [5] for circuit modeling of small surface-mount power beads which are used as inductors in modern switching DC-DC converters. Even an accurate equivalent circuit synthesis method for multi-port networks from frequency response up to $5 \mathrm{GHz}$ has been developed by [6]. The disadvantage of these methods is very long execution time. Implementation of these methods requires many different measurements with many different testfixture structures. This paper describes an extended simple passive network synthesis method, which requires only a simple two-terminal impedance frequency characteristic measurement. Only one test fixture can be used, which is suitable for the described passive network synthesis method.

According to [7], the known techniques to a linear system equivalent calculation in frequency domain are: a resonance frequency matching method, a corrective filter method, a continuous fractional expansion method and an approximate Foster equivalent method. These methods are examples of different iterative direct synthesis techniques. The advantages of direct methods are simplicity and straight forward calculation. This paper describes a modified direct synthesis element-by-element extraction method of approximated passive circuit impedance rational function. This synthesis method based on the element-byelement extraction algorithm in [7] is modified and extended to calculating series and shunt resistors as lossy elements of passive (linear) circuit network.

\section{A Two-Terminal Impedance Fre- quency Characteristic Measuring Concept}

Before the passive circuit network synthesis process from the two-terminal electrical impedance frequency characteristic can start, it is necessary to measure in some way the impedance characteristic of the real passive circuit network. The electrical impedance of two-terminal electrical circuit is generally defined as the total opposition which a circuit offers to the flow of alternating current at a given 
frequency. The impedance frequency characteristic can be measured by an auto balancing bridge method, an RF I-V method or a network analysis method [8].

In this paper, the RF I-V method by an Agilent 4396B vector network analyzer (VNA) in an impedance analyzer mode with an Agilent 43961A RF impedance test adapter is used. An RF coaxial cable or a coaxial line as the RF transmission line has $50 \Omega$ characteristic impedance. It must be noted that the output of the used RF coaxial line is connected to the measured impedance whose value is unknown. The VNA with the RF impedance test adapter must be calibrated to intended measuring terminals (connector) by a standard calibration kit. The calibration minimizes measurement errors caused by the test adapter and the RF coaxial line [9].

\section{Theoretical Approach of Passive Network Synthesis}

The passive network synthesis from the required circuit function, such as the impedance frequency characteristic in this case, is well known subject in the electrical circuit theory. The passive network synthesis is estimation of all passive circuit elements of circuit model which is valid for the required circuit function in a specified range (e.g. frequency range) and conditions (circuit model simplification). The first step of passive network synthesis from the measured impedance frequency characteristic is obtaining the required impedance function which depends on the frequency [10].

The Laplace representation of impedance can be described by a rational function. The polynomials roots of rational impedance function determine the frequency characteristic shape of this function. The general impedance rational function according to [7] and [11] within a scaling constant $K$ is given by the following equation

$$
Z(s)=\frac{N(s)}{D(s)}=\frac{\sum_{k=0}^{n} a_{k} s^{k}}{\sum_{l=0}^{m} b_{l} s^{l}}=K \frac{\prod_{k=1}^{n}\left(s-s_{z, k}\right)}{\prod_{l=1}^{m}\left(s-s_{\mathrm{p}, l}\right)}
$$

where $a_{k}$ is the $k$-th numerator polynomial coefficient, $b_{l}$ is the $l$-th denominator polynomial coefficient, $s_{z, k}$ is the $k$-th position of impedance zero, $s_{\mathrm{p}, l}$ is the $l$-th position of impedance pole in the s-plane and $s$ is the Laplace variable. The Laplace variable $s$ is known as a complex frequency and for a sinusoidal steady state linear circuits calculation (or an $\mathrm{AC}$ analysis) the following substitution according to [12] is used

$$
s=\sigma+\mathrm{j} \omega=0+\mathrm{j} 2 \pi f
$$

where $\sigma$ is the damping factor, $\mathrm{j}$ is the imaginary unit of complex number, $\omega$ is the angular frequency and $f$ is the frequency of excitation (or driving) signal.

As written in (1), the impedance zeros are the roots of the following equation

$$
N(s)=0 \rightarrow \lim _{s \rightarrow \omega_{z, k}} Z(s)=0
$$

and impedance poles are roots of equation

$$
D(s)=0 \rightarrow \lim _{s \rightarrow \omega_{\mathrm{p}, l}} Z(s)=\infty .
$$

All coefficients of polynomials $N(s)$ and $D(s)$ are real, therefore the poles and zeros must be either purely real or appear in complex conjugate pairs. In a case when only purely complex conjugate imaginary root pairs of impedance function polynomials will be considered the impedance function will be in the following form [10]

$$
Z(s)=K \frac{\prod_{k=1}^{\frac{n}{2}}\left(s^{2}+\omega_{2 z, k}^{2}\right)}{\prod_{l=1}^{\frac{m}{2}}\left(s^{2}+\omega_{2 \mathrm{p}, l}^{2}\right)} .
$$

When one pole at the beginning of the $s$-plane is assumed due to a reference circuit element (in the s-plane origin, Section 4 explained why) then the impedance function will be in following form

$$
Z(s)=K \frac{\prod_{k=1}^{\frac{n}{2}}\left(s^{2}+\omega_{2 z, k}^{2}\right)}{s \prod_{l=2}^{\frac{m-1}{2}}\left(s^{2}+\omega_{2 \mathrm{p}, l}^{2}\right)}
$$

where $\omega_{2 z, k}$ is the $k$-th angular series resonance frequency (angular frequency of double zero) of impedance function and $\omega_{2 \mathrm{p}, l}$ is the $l$-th angular parallel resonance frequency (angular frequency of double pole) of the impedance function. The series resonance frequency is reflected as a local impedance minimum with zero impedance phase at this frequency. And the parallel resonance frequency is reflected as a local impedance maximum with zero impedance phase at this frequency [8].

The aim of this paper is adding frequency bandwidths of impedance resonances to obtain lossy elements (resistors and conductors) of passive circuit model for cases with quality factor lower than infinity. For this situation a biquad impedance function with complex conjugate pairs of zeros and poles is taken according to [13]

$$
Z(s)=\frac{s^{2}+\frac{\omega_{\mathrm{z}}}{Q_{\mathrm{z}}} s+\omega_{\mathrm{z}}^{2}}{s^{2}+\frac{\omega_{\mathrm{p}}}{Q_{\mathrm{p}}} s+\omega_{\mathrm{p}}^{2}}
$$

where $\omega_{\mathrm{z}}$ is the angular series resonance frequency, $Q_{\mathrm{z}}$ is the zero quality factor, $\omega_{\mathrm{p}}$ is the angular parallel resonance frequency and $Q_{\mathrm{p}}$ is the pole quality factor of biquad impedance function. By comparing this function with the general quality factor equation according to [14]

$$
Q=\frac{\omega_{0}}{B}
$$

where $Q$ is the bandpass (or resonance) quality factor, $\omega_{0}$ is the center (or resonance) frequency and $B$ is the bandpass (or resonance) frequency bandwidth, the following impedance function with frequency bandwidths of each resonance and one pole at the beginning of the s-plane is obtained 


$$
Z(s)=K \frac{\prod_{k=1}^{\frac{n}{2}}\left(s^{2}+B_{2 z, k} s+\omega_{2 z, k}^{2}\right)}{s \prod_{l=2}^{\frac{m-1}{2}}\left(s^{2}+B_{2 \mathrm{p}, l} s+\omega_{2 \mathrm{p}, l}^{2}\right)}
$$

where $B_{2 \mathrm{z}, k}$ is the $k$-th angular frequency bandwidth of series resonance and $B_{2 \mathrm{p}, l}$ is the $l$-th angular frequency bandwidth of parallel resonance. For passive circuit synthesis, it is necessary to calculate all polynomials coefficients of impedance rational function (1) after substituting resonance frequencies and their bandwidths by numeric values into the impedance function (9). The scaling constant $K$ includes an impedance norm coefficient that is described by the following equation

$$
\begin{gathered}
k_{\mathrm{Z}}=\frac{Z_{\text {norm }}(s)}{Z(s)}, \\
K=\frac{1}{k_{\mathrm{Z}}}
\end{gathered}
$$

where $k_{Z}$ is the impedance norm, $Z_{\text {norm }}$ is the normalized impedance and $Z$ is the original impedance [15].

A numerator polynomial degree $n$ of impedance function (1) may be different from a denominator polynomial degree $m$ by no more than one. If this condition is violated, then the circuit model cannot include passive circuit elements only (resistors, conductors, inductors and capacitors) [7]. The values of angular frequencies can be normalized to the lowest resonance frequency for reducing large numbers in calculations. A software multiplication of polynomials can be done using a simple polynomial multiplication algorithm mentioned for example in [16].

After obtaining all coefficients of rational impedance function (1) and fulfillment the passive circuit elements condition, the next step is a passive network elements extraction. In [7] a nice algorithm is shown how to extract simple circuit elements from the impedance function. This algorithm is extended to series and shunt resistors (lossy elements) calculation. The proposed element-by-element extraction algorithm is shown in Appendix A.

The polynomials division is used for the impedance elements calculation which brings six considered solutions from all possible solutions summarized in Tab. 1 .

The $L_{\mathrm{x}}, R_{\mathrm{x}}, C_{\mathrm{x}}$ and $G_{\mathrm{x}}$ are values of inductor, resistor, capacitor and conductor (shunt resistor with $1 / G_{\mathrm{x}}$ value) respectively. The first extracted element is always $Z(s)$ due to the chosen reference circuit element which is an RF series coupling capacitor for this case. Therefore, the first element can be the $C_{\text {Series }}$ capacitor or the $L_{\text {Series }}$ inductor. It must be noted that the type of the first calculated element is undefined in the proposed extraction algorithm. Then the $C_{\text {Series }}$ or the $L_{\text {Series }}$ element, which is the $Z(s)$ type, is followed by the $R_{\text {Series }}$ resistor element. The residual polynomial is calculated after each polynomial division. The pro-

\begin{tabular}{|c|c|c|c|}
\hline Element & $Z_{x}(s)$ or $Y_{x}(s)$ & $\begin{array}{l}\text { Conditions of } \\
Z(s) \text { function }\end{array}$ & Element value \\
\hline$\stackrel{R_{\text {Series }}}{\longleftarrow}$ & $Z_{x}(s)=R_{\text {Series }}$ & $\begin{array}{c}n=m \\
\text { and previous } \\
\text { element is } Z(s) \\
\text { type }\end{array}$ & $R_{\text {Series }}=\frac{N[n]}{D[m]}$ \\
\hline$\prod G_{\text {shunt }}$ & $Y_{x}(s)=G_{\text {Shunt }}$ & $\begin{array}{c}n=m \\
\text { and previous } \\
\text { element is } Y(s) \\
\text { type }\end{array}$ & $G_{\text {Shunt }}=\frac{D[m]}{N[n]}$ \\
\hline$\overbrace{}^{L_{\text {Series }}}$ & $Z_{x}(s)=s L_{\text {Series }}$ & $n>m$ & $L_{\text {Series }}=\frac{N[n]}{D[m]}$ \\
\hline $\mathrm{C}_{\text {Series }}$ & $Z_{x}(s)=\frac{1}{s C_{\text {Series }}}$ & $\begin{array}{c}N[0]>0 \text { and } \\
D[0]=0\end{array}$ & $C_{\text {Series }}=\frac{D[1]}{N[0]}$ \\
\hline$\left\{L_{\text {Shunt }}\right.$ & $Y_{x}(s)=\frac{1}{s L_{\text {Shunt }}}$ & $\begin{array}{c}N[0]=0 \text { and } \\
D[0]>0\end{array}$ & $L_{\text {Shunt }}=\frac{N[1]}{D[0]}$ \\
\hline$\frac{1}{T} c_{\text {shunt }}$ & $Y_{x}(s)=s C_{\text {Shunt }}$ & $n<m$ & $C_{\text {Shunt }}=\frac{D[m]}{N[n]}$ \\
\hline \multicolumn{4}{|c|}{$Z(s)=\frac{N(s)}{D(s)}=\frac{N[0]+N[1] s+N[2] s^{2}+\ldots+N[n] s^{n}}{D[0]+D[1] s+D[2] s^{2}+\ldots+D[m] s^{m}}$} \\
\hline
\end{tabular}
posed element extraction sequence with its conditions was chosen and it is based on the extraction algorithm in [7].
Tab. 1. Simple circuit element extraction conditions and calculations (the first step of extraction for this case, for the next step there is residual polynomial after polynomials division).

The following equations (12-14) show examples of impedance rational function polynomials divisions (without resonance bandwidths for clarity), where $\operatorname{Res}_{x}(s)$ is the residual polynomial.

$$
\begin{gathered}
Z(s)=\frac{N(s)}{D(s)}=Z_{1}(s)+\frac{\operatorname{Res}_{1}(s)}{D(s)}, \\
\frac{D(s)}{\operatorname{Res}_{1}(s)}=Y_{1}(s)+\frac{\operatorname{Res}_{2}(s)}{\operatorname{Res}_{1}(s)} \\
\frac{\operatorname{Res}_{1}(s)}{\operatorname{Res}_{2}(s)}=Z_{2}(s)+\frac{\operatorname{Res}_{3}(s)}{\operatorname{Res}_{2}(s)}
\end{gathered}
$$

The condition for performing the next polynomials division is the positive real residual function after the current division. If the residual function is negative, then the impedance rational function cannot be assembled from the passive circuit elements in the Cauer passive network model form. On the other hand, negative resistors in the mathematical circuit model can be considered because this model can be used for the computer simulation only. If the above polynomials division steps will be applied, then the division results can be written into the following continuous fraction expansion

$$
Z(s)=Z_{1}(s)+\frac{1}{Y_{1}(s)+\frac{1}{Z_{2}(s)+\ldots}} .
$$


a)

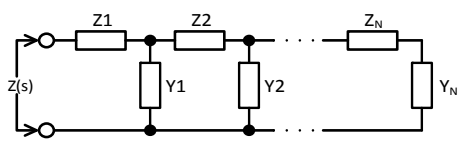

b)

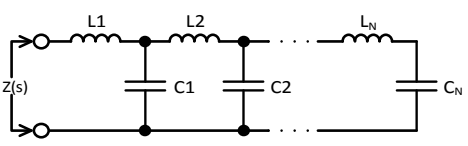

c)

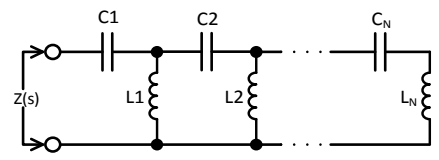

Fig. 1. The Cauer passive network impedance models: a) general model, b) first Cauer and c) second Cauer canonical form.

This ladder equation according to [17] has passive network realizations called the first and second Cauer canonical form which are shown in Fig. 1.

\section{Practical Approach of Passive Network Synthesis}

This section explains the practical approach of successful passive network synthesis using the proposed element-by-element extraction method from the impedance function. It must be noted that all of measured impedance function elements are unknown except the one element that is always presented in the measurement setup. This element is the RF input coupling capacitor which creates the one impedance pole at the beginning of $s$-plane. The first series capacitor of synthesized passive network is the RF coupling capacitor and its value is used as the reference value for calculation of other circuit model elements. The RF coupling capacitor value can be measured by an electronic impedance meter like an LCR meter with four terminal-pair definition [18]. Figure 2 shows one realization example of a measured impedance function without lossy elements.

The impedance norm coefficient (10) is calculated from the measured RF coupling capacitor value and the first series capacitor of the synthesized circuit model as

$$
k_{\mathrm{Z}}=\frac{C_{\mathrm{C}}}{c_{\text {series } 1}}
$$

where $k_{Z}$ is the impedance norm coefficient, $C_{\mathrm{C}}$ is the RF coupling capacitor value and $c_{\text {series } 1}$ is the first series capacitor value of the synthesized circuit model. The real values of the synthesized circuit model elements are calculated by the following equations

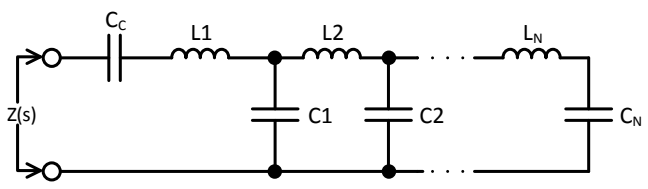

Fig. 2. The example of passive network impedance model with the known coupling capacitor $C_{\mathrm{C}}$.

$$
\begin{gathered}
L=\frac{l}{k_{\mathrm{Z}}}, \\
C=k_{\mathrm{Z}} c, \\
R=\frac{r}{k_{\mathrm{Z}}}
\end{gathered}
$$

where the $L, C, R$ are real values of inductors, capacitors, resistors and the $l, c, r$ are normalized values of inductors, capacitors and resistors from the synthesized circuit model with the impedance norm coefficient $k_{\mathrm{Z}}$.

A reference ideal passive circuit model with three impedance resonances has been introduced for verification of the proposed synthesis method. The reference circuit model is shown in Fig. 3.

Table 2 shows the values of resonance frequencies and resonance bandwidths of the reference circuit model, where $f_{\text {ser res }}$ is the series resonance frequency, $B_{\text {ser res }}$ is the series resonance bandwidth, $f_{\text {par res }}$ is the parallel resonance frequency and $B_{\text {par_es }}$ is the parallel resonance bandwidth. These values were obtained by an $\mathrm{AC}$ simulation of the reference model in the SPICE circuit simulator.

The synthesized circuit model has circuit elements calculated by the proposed element-by-element extraction method from parameters in Tab. 2. This circuit model has the same circuit topology as the reference circuit model from Fig. 3. The extracted elements values and their relative error $\left(\delta_{\text {synthesis }}\right)$ are shown in Tab. 3.

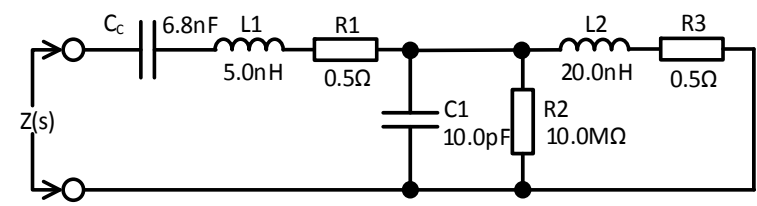

Fig. 3. The reference circuit model for the impedance synthesis method verification.

\begin{tabular}{|l|l|l|l|}
\hline $\boldsymbol{f}_{\text {ser_res }}[\mathbf{M H z}]$ & $\boldsymbol{B}_{\text {ser_res }}[\mathbf{M H z}]$ & $\boldsymbol{f}_{\text {par_res }}[\mathbf{M H z}]$ & $\boldsymbol{B}_{\text {par_res }}[\mathbf{M H z}]$ \\
\hline 12.20064 & 6.37313 & 355.87286 & 4.01481 \\
\hline 796.17750 & 13.56498 & none & none \\
\hline
\end{tabular}

Tab. 2. The reference circuit model impedance resonance frequencies and bandwidths (rounded to five decimal places).

\begin{tabular}{|l|l|l|l|}
\hline Model element & Reference & Synthesis result & $\boldsymbol{\delta}_{\text {Synthesis }}[\%]$ \\
\hline $\mathrm{C} 1$ & $6.800 \mathrm{nF}$ & $6.800 \mathrm{nF}$ & 0.000 \\
\hline $\mathrm{L} 1$ & $5.000 \mathrm{nH}$ & $4.999 \mathrm{nH}$ & -0.020 \\
\hline $\mathrm{R} 1$ & $0.500 \Omega$ & $0.500 \Omega$ & 0.000 \\
\hline $\mathrm{C} 2$ & $10.000 \mathrm{pF}$ & $9.999 \mathrm{pF}$ & -0.010 \\
\hline $\mathrm{R} 2$ & $10.000 \mathrm{M} \Omega$ & $0.678 \mathrm{M} \Omega$ & -93.220 \\
\hline $\mathrm{L} 2$ & $20.000 \mathrm{nH}$ & $20.002 \mathrm{nH}$ & 0.010 \\
\hline $\mathrm{R} 3$ & $0.500 \Omega$ & $0.502 \Omega$ & 0.400 \\
\hline
\end{tabular}

Tab. 3. The reference circuit model synthesis results (rounded to three decimal places). 


\begin{tabular}{|c|c|c|c|}
\hline$\Delta \boldsymbol{B}_{\text {ser_res }}[\mathrm{Hz}]$ & $\Delta \boldsymbol{R}_{\text {Series }}[\boldsymbol{\Omega}]$ & $\Delta \boldsymbol{B}_{\text {par_res }}[\mathrm{Hz}]$ & $\Delta \boldsymbol{R}_{\text {Shunt }}[\boldsymbol{\Omega}]$ \\
\hline 1 & $0.1 \mu$ & 1 & 21.721 \\
\hline 10 & $0.4 \mu$ & 10 & 217.272 \\
\hline
\end{tabular}

Tab. 4. The basic sensitivity analysis of the proposed extraction method on resonance bandwidths.

The proposed synthesis method shows very good result but there is a problem with high values of shunt resistors because the proposed extraction method has high sensitivity on parallel resonance bandwidth value. The high shunt resistor value causes very narrowband parallel resonance bandwidth which cannot be easy precisely determined. The proposed extraction method basic sensitivity results on resonance bandwidth change is shown in Tab. 4.

The impedance characteristics vs frequency as the $\mathrm{AC}$ simulation results of reference and synthesized circuit model from the SPICE circuit simulator are shown in Fig. 4 and 5. Figure 6 shows a graphical user interface of the impedance passive network synthesizer software which was written in $\mathrm{C \#}$ for fast passive circuit elements calculation using the proposed element-by-element extraction method. This software uses all methods which are described in this paper. Figure 7 shows the example of real impedance circuit which is used as a basic EMC power supply filter for a basic EMC DPI testing. This circuit is used for the proposed synthesis method verification in the real world. The $4.7 \mathrm{nF}$ RF coupling capacitor value was selected according to the RF DPI immunity test method which is used for the EMS test at IC level [1]. The basic EMC filter schematic diagram is shown in Fig. 8.

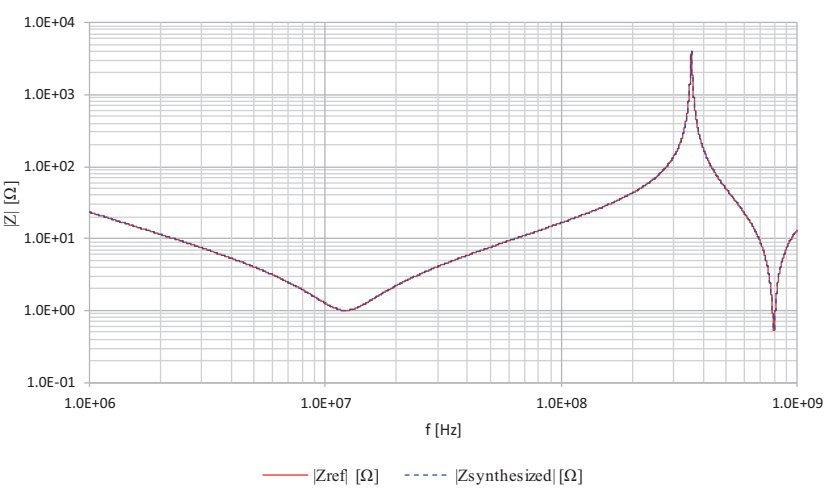

Fig. 4. The impedance magnitude characteristics of reference and synthesized circuit model.

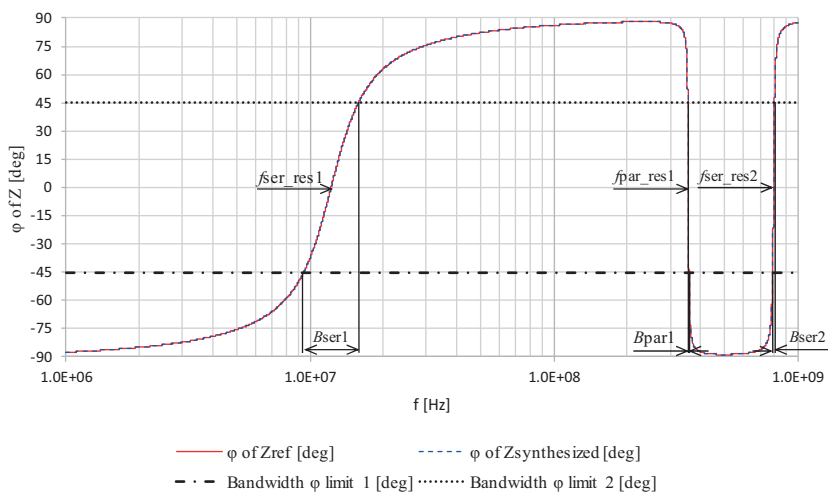

Fig. 5. The impedance phase characteristics of reference and synthesized circuit model.

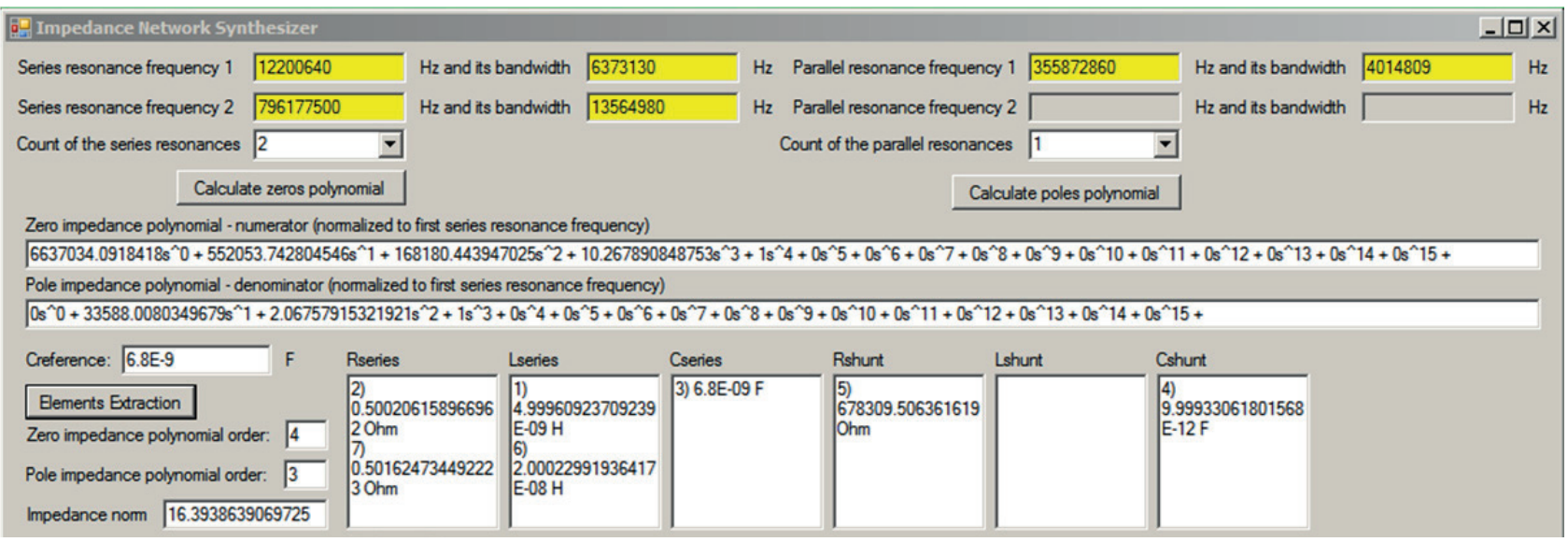

Fig. 6. The passive network impedance synthesizer software using the proposed element-by-element extraction method (results are rounded to appropriate decimal places).

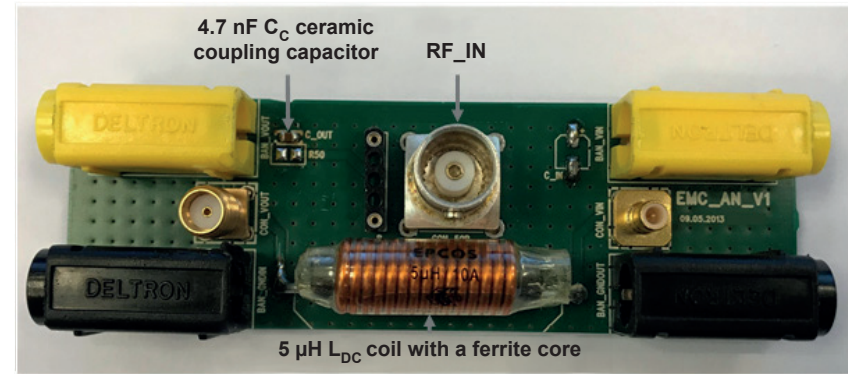

Fig. 7. Example of real impedance circuit (the basic EMC filter for basic EMC DPI test).

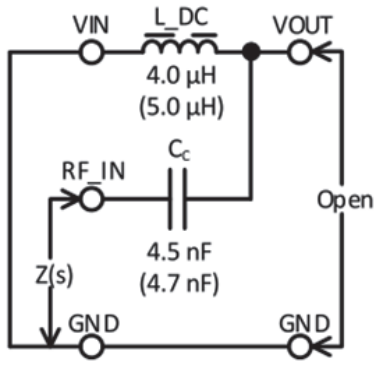

Fig. 8. Schematic diagram of real impedance circuit example (the basic EMC filter for basic EMC DPI test). 
The basic EMC filter high frequency impedance was measured at high frequency input (RF_IN) for case when the DC power supply input (VIN) connector is short to ground with very low impedance (by very short conductor). Then the resonance frequencies and bandwidths were extracted from the measured impedance characteristic vs frequency. The synthesized passive circuit model from these parameters is shown in Fig. 9.

Table 5 shows values of extracted resonance frequencies and resonance bandwidths from the measured impedance characteristic of basic EMC filter, where $f_{\text {ser res }}$ is series resonance frequency, $B_{\text {ser_res }}$ is series resonance bandwidth, $f_{\text {par_res }}$ is parallel resonance frequency and $B_{\text {par_es }}$ is parallel resonance bandwidth. The synthesized circuit model elements value and description are summarized in Tab. 6 .

The impedance characteristics vs frequency as the impedance measurement and $\mathrm{AC}$ simulation results of basic EMC filter real circuit and its synthesized circuit model from the SPICE circuit simulator are shown in Fig. 10 and 11.

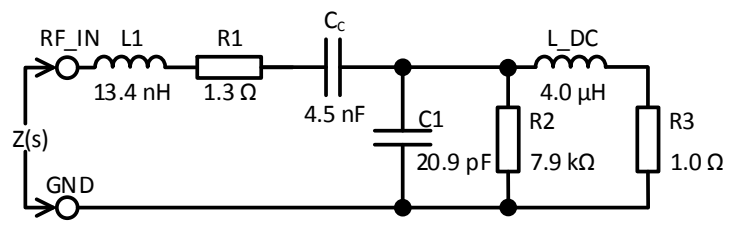

Fig. 9. The synthesized passive circuit model of basic EMC filter.

\begin{tabular}{|c|c|c|c|}
\hline $\boldsymbol{f}_{\text {ser_res }}[\mathbf{M H z}]$ & $\boldsymbol{B}_{\text {ser_res }}[\mathbf{M H z}]$ & $\boldsymbol{f}_{\text {par_res }}[\mathbf{M H z}]$ & $\boldsymbol{B}_{\text {par_res }}[\mathbf{M H z}]$ \\
\hline 1.17968 & 0.09662 & 17.38654 & 1.01143 \\
\hline 302.02947 & 16.14057 & Not considered & Not considered \\
\hline
\end{tabular}

Tab. 5. The RF_IN basic EMC filter impedance resonance frequencies and bandwidths with the V_IN shorted to the ground (rounded to five decimal places).

\begin{tabular}{|l|l|l|}
\hline Model element & Value & \multicolumn{1}{c|}{ Description } \\
\hline L1 & $13.4 \mathrm{nH}$ & $\begin{array}{l}\text { The inductance of RF } \\
\text { connector, PCB track and } \\
\text { coupling capacitor. }\end{array}$ \\
\hline R1 & $1.3 \Omega$ & $\begin{array}{l}\text { The DC resistance of RF } \\
\text { connector, PCB track and } \\
\text { coupling capacitor. }\end{array}$ \\
\hline C $_{\text {C }}$ & $4.5 \mathrm{nF}$ & $\begin{array}{l}\text { The coupling capacitor capacity } \\
\text { (verified by the LCR meter). }\end{array}$ \\
\hline C1 & $20.9 \mathrm{pF}$ & $\begin{array}{l}\text { The interturn capacity of DC } \\
\text { coil with the PCB track capacity } \\
\text { (to the ground). }\end{array}$ \\
\hline R2 & $7.9 \mathrm{k} \Omega$ & $\begin{array}{l}\text { The ferrite core loss resistance } \\
\text { of DC coil. }\end{array}$ \\
\hline L_DC & $4.0 \mu \mathrm{H}$ & $\begin{array}{l}\text { The inductance of DC coil } \\
\text { (verified by the LCR meter). }\end{array}$ \\
\hline R3 & $1.0 \Omega$ & $\begin{array}{l}\text { The winding resistance of DC } \\
\text { coil and short to the ground. }\end{array}$ \\
\hline
\end{tabular}

Tab. 6. The synthesized circuit model elements of basic EMC filter (rounded to one decimal place).

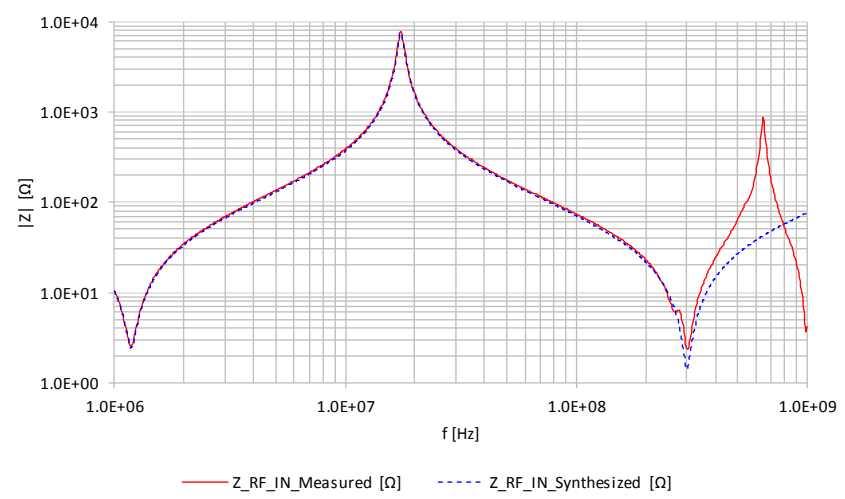

Fig. 10. The impedance magnitude characteristics of basic EMC filter and its synthesized circuit model.

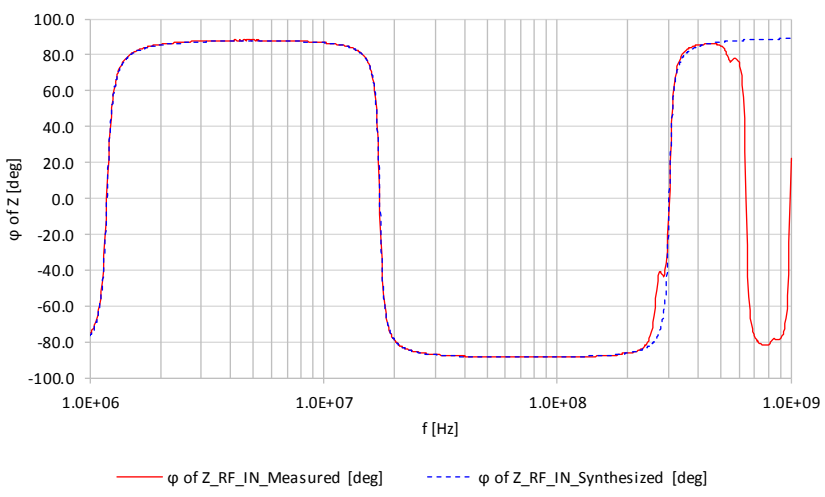

Fig. 11. The impedance phase characteristics of basic EMC filter and its synthesized circuit model.

The basic EMC filter circuit model is built of frequency-independent elements and the simulated results show very good correlation with measured results in frequency range from 1 to $200 \mathrm{MHz}$. The synthesized circuit model is a simplified finite lumped approximation for distributed parameter circuit system. Therefore, the correlation at last considered resonance frequency is poor. From the impedance characteristic, there is an apparent limitation of the proposed synthesis method. It is impossible to obtain all input parameters for the synthesis method when the resonances are very close to each other. The very close measured resonances are seen in Fig. 11 from $300 \mathrm{MHz}$ approximately. But if a certain inaccuracy of the model is considered, a simple approximation can be used for the resonance bandwidth determination as described in the following equations.

$$
\begin{gathered}
f_{1}=\frac{f_{0}^{2}}{f_{2}}, \\
B=f_{2}-f_{1}
\end{gathered}
$$

where $f_{0}, f_{1}$ and $f_{2}$ are the resonance frequency, the first and second frequency which determine $B$ resonance bandwidth. For this approximation it is necessary to know at least one boundary and resonant frequencies of considered resonance bandwidth [19]. 


\section{Conclusion}

The aim of this article was to describe and show a practical usability of the proposed extended passive network synthesis. This synthesis uses element-by-element extraction method which was extended to series and shunt resistors calculation as lossy elements of passive circuit network. This method was verified on the ideal reference circuit model and on the real impedance circuit with very good result. An accurate lumped-element circuit model of the basic EMC filter is extracted. This model is valid in the considered frequency range from 1 to $200 \mathrm{MHz}$. The passive network synthesis can be used for estimation of load impedance model of ICpins, for more accurate passive circuit simulation model, for estimation of impedance model of PCB tracks with passive circuit components etc. The proposed impedance passive network synthesizer software using the described element-by-element extraction method was presented. The proposed synthesis method is accurate, fast and does not need many special measurements for input parameters of this method. However, there are some limitations as well as accuracy of the shunt resistor (conductivity) calculation due to high sensitivity on correct parallel resonance bandwidth value and usability on circuits with clear resonances. This method can be improved for other possible circuit model variations.

\section{References}

[1] IEC. IEC62132-4: Integrated Circuits - Measurement of Electromagnetic Immunity $150 \mathrm{kHz}$ to $1 \mathrm{GHz}$ - Part 4: Direct $\mathrm{RF}$ Power Injection Method. 2006.

[2] BOTT, R., DUFFIN, R. J. Impedance synthesis without use of transformers. Journal of Applied Physics, 1949, vol. 20, no. 8, p. 816-816. DOI: $10.1063 / 1.1698532$

[3] KELLY, P. M. A unified approach to two-terminal network synthesis. IRE Transactions on Circuit Theory, 1961, vol. 8, no. 2 , p. 153-164. DOI: 10.1109/TCT.1961.1086763

[4] LEE, J. A., KIM, D., EO, Y. Circuit modelling of multi-layer ceramic capacitors using S-parameter measurements. In International SoC Design Conference. Busan (South Korea), 2008, vol. 1, p. I-358-I-361. DOI: 10.1109/SOCDC.2008.4815646

[5] BAČMAGA, J., BLEČIĆ, R., GILLON, R., BRIĆ, A. Highfrequency model of a setup for time-domain inductor characterization. In 2018 International Symposium on Electromagnetic Compatibility (EMCEUROPE). 2018, p. 590-595. DOI: 10.1109/EMCEurope.2018.8485154

[6] AVULA, V., ZADEHGOL, A. A novel method for equivalent circuit synthesis from frequency response of multiport network. In Proc. of the 2016 International Symposium on Electromagnetic Compatibility - EMC EUROPE 2016. Wroclaw (Poland), 2016. DOI: 10.1109/EMCEurope.2016.7739270

[7] MOHAMED, S., SALAMA, M. M. A., MANSOUR, R. Automated network synthesis utilizing MAPLE. In The 46th Midwest Symposium on Circuits and Systems. 2003, vol. 3, p. 1179-1184. DOI: 10.1109/MWSCAS.2003.1562504

[8] KEYSIGHT TECHNOLOGIES, USA. Impedance Measurement Handbook: A Guide to Measurement Technology and Techniques. $6^{\text {th }}$ ed. (application note). 140 pages. [Online] Cited 2018-09-08. Available at:

https://iterature.cdn.keysight.com/litweb/pdf/5950-3000.pdf
[9] PIROLA, M., TEPPATI, V., CAMARCHIA, V. Microwave measurements. Part I: Linear measurements. IEEE Instrumentation and Measurement Magazine, 2007, vol. 10, no. 2, p. 14-19. DOI: 10.1109/MIM.2007.364959

[10] MORCHED, A. S., KUNDUR, P., Identification and modelling of load characteristics at high frequencies. IEEE Transactions on Power Systems, 1987, vol. 2, no. 1, p. 153-159. DOI: 10.1109/TPWRS.1987.4335091

[11] WILliAMS, A. B., TAYLOR, F. J. Electronic Filter Design Handbook. Chapter 1: Introduction to modern network theory, p. 1-8. 4th ed. London: McGraw-Hill, 2006. ISBN: 0-07-147171-5

[12] NEWCOMB, R. W. Analysis in the time domain. Chapter 25 in CHEN, W. K. (ed.) The Circuits and Filters Handbook, p. 783-799. 2nd ed. Boca Raton, FL: CRC Press, 2003. ISBN: 08493-0912-3

[13] ANTONIOU, A. General characteristics of filters. Chapter 69 in CHEN, W. K. (ed.) The Circuits and Filters Handbook, p. 2199 2226. 2nd ed. Boca Raton, FL: CRC Press, 2003. ISBN: 0-84930912-3

[14] SU, K. Analog Filters. (Chapter 4: Frequency transformation, p. 77-88.) 2nd ed. Norwell: Kluwer Academic Publishers, 2002. ISBN: 0-4020-7033-0

[15] SU, K. Analog Filters. (Chapter 1.6: Normalization and denormalization - scaling, p. 13-16.) 2nd ed. Norwell: Kluwer Academic Publishers, 2002. ISBN: 0-4020-7033-0

[16] CORMEN, T. H., LEISERSON, CH. E., RIVEST, R. L., STEIN, C. Introduction to Algorithms. (Chapter 30: Polynomials and the FFT, p. 898-925.) 3rd ed. Cambridge, Mass.: MIT Press, 2009. ISBN: 978-0-262-53305-8

[17] CHEN, W. K. Synthesis of LCM and RC one-port networks. Chapter 75 in CHEN, W. K. (ed.) The Circuits and Filters Handbook, p. 2327-2337. 2nd ed., Boca Raton, FL: CRC Press, 2003. ISBN: 0-8493-0912-3

[18] CALLEGARO, L. Traceable measurements of electrical impedance. IEEE Instrumentation and Measurement Magazine, 2015, vol. 18, no. 6, p. 42-46. DOI: 10.1109/MIM.2015.7335839

[19] DAVIS, M. A. Approximation. Chapter 70 in CHEN, W. K. (ed.) The Circuits and Filters Handbook, p. 2227-2257. 2nd ed., Boca Raton, FL: CRC Press, 2003. ISBN: 0-8493-0912-3

\section{About the Authors ...}

David KROLÁK was born in Frýdek-Místek, Czech Republic. He received his B.Sc. and M.Sc. degree in Electronics and Communication from the Dept. of Radio Electronics, Brno University of Technology, Czech Republic, in 2014 and 2016, respectively. He is currently a student of combined doctoral study in the Electronics and Communications program of same department. From 2016 he is working at ON Semiconductor as an EMC test engineer. His interests include an analog IC design for automotive products and electrical measurements with focus on EMC at IC level.

Pavel HORSKÝ received the M.Sc. degree in 1994 in Radio Electronics and the Ph.D. degree in 1998 in the field of metrology at the Brno University of Technology, Czech Republic. In 2011 he became an associated professor at the same university. He is teaching analog design courses for Ph.D. students. From 1997 he works as an analog and 
mixed-signal design engineer, technical project leader and leader of analog design group at Alcatel Microelectronics, AMI Semiconductor and ON Semiconductor. Currently he is a member of technical staff at ON Semiconductor. His professional interests include an analog / mixed-signal IC design for automotive products with focus on EMC, ESD and reliability. Dr. Horský is an author and co-author of $60+$ publications and 16 issued US patents.

\section{Appendix A}

The proposed element-by-element extraction method flow chart.

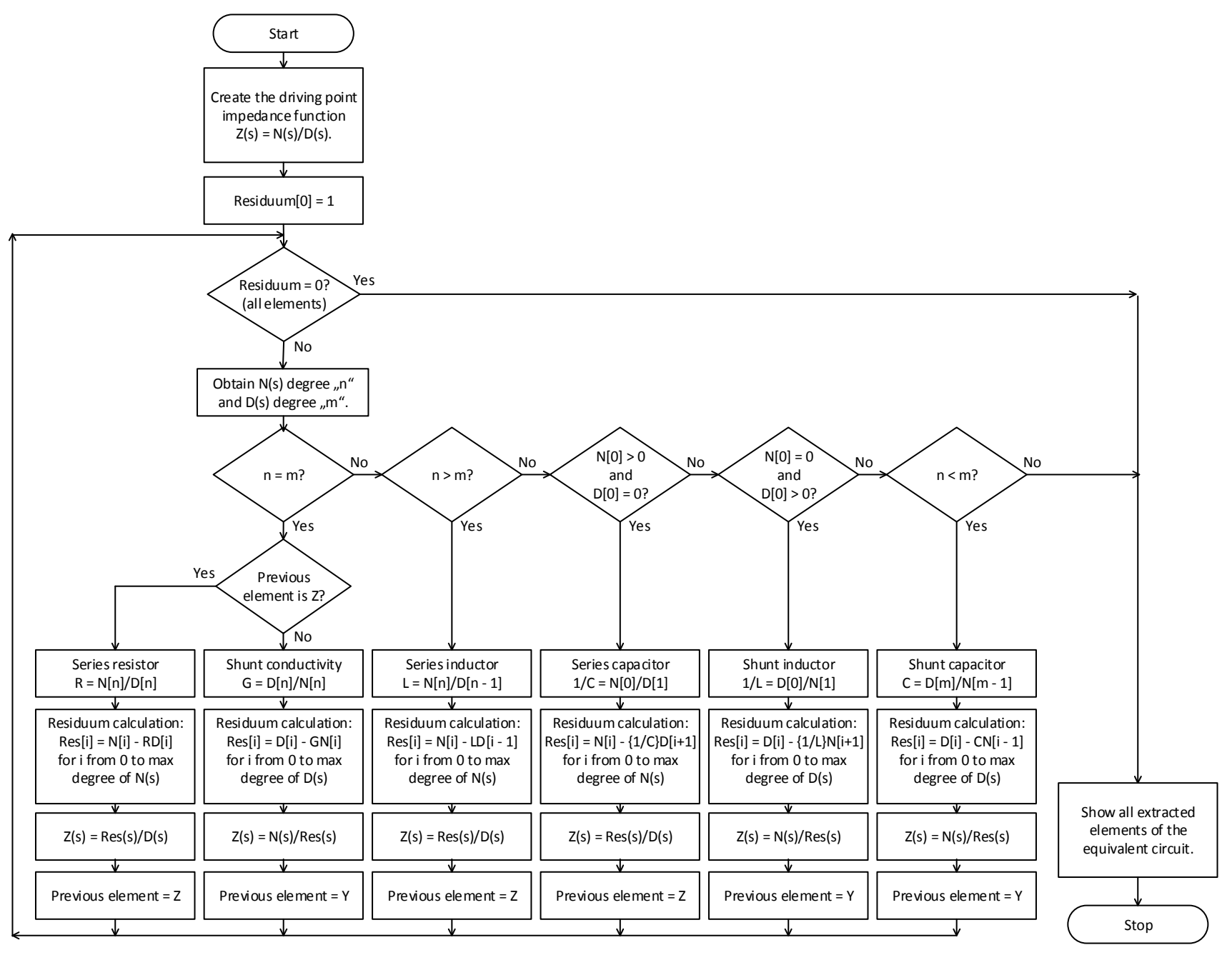

